\title{
Multi-mJ mid-IR light bullets in air
}

\author{
Valentina Shumakova ${ }^{1}$, Skirmantas Ališauskas ${ }^{1}$, Andrius Baltuška ${ }^{1,2}$, Pavel Malevich ${ }^{1}$, \\ Alexander Voronin ${ }^{3,4}$, Alexander Mitrofanov ${ }^{3,4}$, Dmitriy Sidorov-Biryukov ${ }^{3,4}$, Aleksey \\ Zheltikov ${ }^{3,4,5}$, Daniil Kartashov', Audrius Pugžlys ${ }^{1,2, *}$ \\ ${ }^{1}$ Photonics Institute, TU Wien, Gusshausstrasse 27-387, A-1040 Vienna, Austria \\ ${ }^{2}$ Center for Physical Sciences \& Technology, Savanoriu Ave. 231 LT-02300 Vilnius, Lithuania \\ ${ }^{3}$ Physics Department, M.V. Lomonosov Moscow State University, 119992 Moscow, Russia \\ ${ }^{4}$ Russian Quantum Center, ul. Novaya 100, Skolkovo, Moscow Region, 143025 Russia \\ ${ }^{5}$ Dep. of Physics and Astronomy, Texas A\&M University, College Station TX, 77843-4242, USA \\ ${ }^{6}$ Friedrich-Schiller University Jena, Max-Wien Platz 1, 07743 Jena, Germany
}

\begin{abstract}
We examine mid-IR light bullets generated in ambient air. 2optical cycle pulses confined in space are generated in filamentation regime. Few-fold solitonic self-compression is achieved for strongly chirped mid-IR pulses.
\end{abstract}

\section{Introduction}

A unique combination of high transparency and anomalous dispersion of Earth atmosphere in the mid-IR spectral range promotes an opportunity for lossless high-energy ultrashort pulse delivery and simultaneous spatio-temporal self-compression, what potentially allows generation on the target of supercontinuum, intense $\mathrm{THz}$ radiation and higher order harmonics. However, multiple molecular resonances present in mid-IR "fingerprint" spectral region are not only providing anomalous dispersion windows, but also complicate propagation dynamics and cause absorption losses, which might be dramatically enhanced via nonlinear spectral broadening. Anomalous dispersion of air between 3.6-4.2 $\mu \mathrm{m}$ [1], makes feasible propagation and self-compression of mid-IR pulses in a solitonic regime [2], [3] and even, as predicted theoretically, formation of light bullets [4], which are ultrashort highly localized light structures propagating without changing their size and shape [5].

Recently we have demonstrated experimentally, that filamentation of mid-IR pulses in air is possible in a presence of rather low density plasma [6], when intensity inside the filament is arrested by the Raman Enhanced Absorption Losses (REAL) caused by the stimulated rotational Raman scattering (SRRS) induced frequency downshift and absorption of the newly generated components by $\mathrm{CO}_{2}$. Since SRRS governed spectral dynamics is highly sensitive to the pulse duration [7], a chirping of mid-IR pulses provides control over the spectro-temporal dynamics and, consequently, over the REAL and pulse energy. Controlling the amount of $\mathrm{CO}_{2}$, which is feasible at laboratory conditions, one can act on the dispersion and on the influence of REAL as well. Altogether, it potentially allows a targeted delivery of the ultrashort multi-mJ mid-IR pulses with an arbitrary pulse energy.

\footnotetext{
*Corresponding author: pugzlys@,tuwien.ac.at
} 
Here we report on the first, to the best of our knowledge, experimental observation of the mid-IR light bullets in ambient air. We show that through the self-compression in filaments pulses with a duration of $30 \mathrm{fs}$ in a round and symmetric beam can be generated on the target.
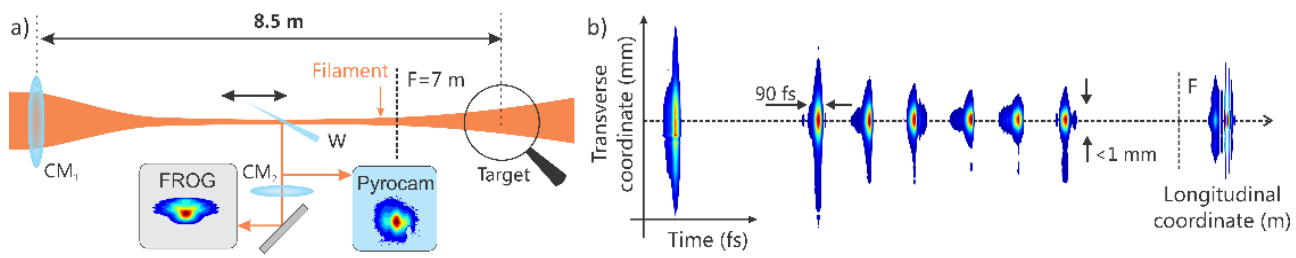

Fig. 1. a) Schematics of the experimental setup: $\mathrm{CM} 1$ - focusing optics with $\mathrm{f}=7 \mathrm{~m}, \mathrm{~W}-\mathrm{CaF} 2$-wedge, CM2 - reimaging optics, FROG - second harmonic generation frequency resolved optical gating apparatus based on AGS NLO crystal, Pyrocam - pyroelectric camera, Target - a desirable "delivery" point, in this particular case corresponding to the $8.5 \mathrm{~m}$ distance from CM1; b) Spatio-temporal evolution along the filament retrieved from experimentally measured FROG traces and beam profiles.

\section{Formation of Light Bullets}

In order to confirm formation of the light bullets, we examined along the propagation the temporal and spatial characteristics of light structures formed during filamentation of 3.9- $\mu \mathrm{m}$ $30-\mathrm{mJ}$ pulses focused with a $f=7 \mathrm{~m}$ spherical mirror (Fig.1 (a)). Results of the measurements reveal that initially 130 -fs positively chirped pulses self-compress to $90 \mathrm{fs}$ at the distance of $3.8 \mathrm{~m}$ from the focusing mirror and keep their temporal shape and duration over the next $2 \mathrm{~m}$ until pulses split of due to accumulated higher-order phase. Sharp trailing edge observed in all temporal profiles along the filament (Fig.1 (b)) occurs due to the self-steepening, as oscillations behind are due to uncompensated higher order phase [8]. Together with the selfcompression initially elliptical beam with $r_{x}=4.5 \mathrm{~mm}, r_{y}=5.5 \mathrm{~mm}$ is highly localized in space in a round symmetrical mode with the diameter of less than $1 \mathrm{~mm}$ (Fig.1 (b)).
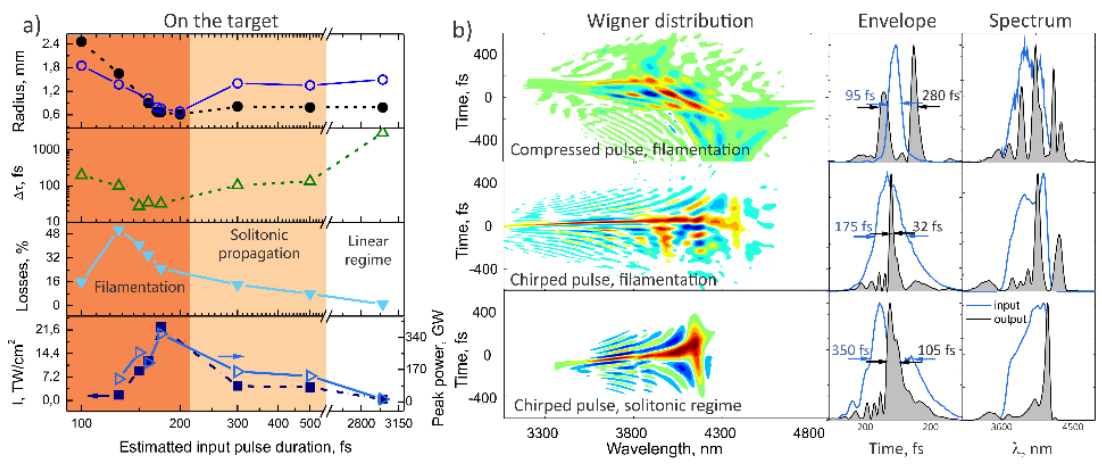

Fig. 2. a) Dependences of pulse/beam output parameters (at the "Target" position) on the initial pulse duration; b) Wigner distributions, time envelopes and spectra of pulses before (blue line) and after the filament (black line) retrieved from SHG FROG traces measured at the "Target" position.

\section{Self-compression in Air}

An introduction of additional initial positive temporal chirp $C>0$ leads to postponed onset of the filamentation due to the lower input peak power $\mathrm{P}=\frac{\mathrm{E}}{1.665 \tau_{0} \sqrt{1+\mathrm{C}^{2}}}$ and results in 
a smaller shift of nonlinear focus position. Consequently, the distance at which chirped pulse attains its transform-limited (TL) duration due to the compensation of the chirp C by the medium having group velocity dispersion (GVD) of opposite sign is:

$$
z_{\min }=\left[\frac{|C|}{1+C^{2}}\right] \frac{\Delta \tau_{0}^{2}}{4 k^{\prime \prime} \ln 2}
$$

where $k^{\prime \prime}$ is the GVD, $\Delta \tau_{0}$ is the TL pulse duration. At the same time the chirping influences the SRRS-induced frequency downshift rates, scaling as $\sim \Delta \tau_{0}^{-3}$ for non-solitonic and $\sim \Delta \tau_{0}^{-4}$ for solitonic pulses [7]. Here the frequency downshift plays a double role, being responsible for an increase of the dispersion [1] and, at the same time, for the energy losses (REAL) (Fig. 2 (a)). When filaments are ignited by the optimally compressed (90-fs) pulses, the amount of losses is significantly lower, than in the case of slightly positive chirped 130 -fs pulses, what can be explained by a dispersion spreading of 90 -fs pulses before the onset of filamentation.

In the Fig.2a the results of complicated interplay between the GVD, SRRS and REAL are shown: in the case of initially stretched pulses with durations ranging from 150 to $200 \mathrm{fs}$. At the distance of $8.5 \mathrm{~m}$ from the focusing mirror an efficient self-compression down to $28 \mathrm{fs}$ can be achieved. At the same time, the energy losses lower more than 2 times from the maximum value of $48 \%$ to $\sim 20 \%$. In addition, initially elliptical beam is self-symmetrized resulting in a round spot with the diameter of $\sim 1 \mathrm{~mm}$, what is a robust sign of filamentation. All together this leads to a formation of light bullet and targeted delivery of high intensity light. In case of shorter, 90-130 fs, initial pulses a beam "on the target" nucleates in the direction of initial ellipticity.

Chirping of the $30-\mathrm{mJ}$ pulses to durations longer than $\sim 300$ fs leads to an abortion of the filamentation due to the drop of peak power below the critical power of self-focusing and yields to propagation of initially elliptical beam without symmetrization. In this case, the solitonic self-compression without filamentation takes place [3].

\section{Conclusions}

We experimentally confirm an existence of theoretically predicted mid-IR light bullets. A generation of 2-cycle mid-IR waveforms is possible at a given distance from the focusing mirror via adjustment of temporal chirp of the filamenting $30-\mathrm{mJ}$ pulses. This is potentially applicable for remote spectroscopy and high-energy beam delivery.

\section{References}

1. Voronin, A. and A. Zheltikov, Scientific Reports 7, 46111 (2017)

2. Voronin, A. and A. Zheltikov, PHYSICAL REVIEW A, 95 (023826) (2017).

3. Mitrofanov A., et al, Optica 4, 1405-1408 (2017)

4. Panagiotopoulos, P., et al., Nat Photon, 9(8): 543-548 (2015)

5. Silberberg Ya., Opt. Lett. 15, 1282-1284 (1990)

6. Shumakova V. et al., CLEO, OSA Technical Digest (online) (2017) paper FM1F.5.

7. Zheltikov, A.M., et al, Phys.-Usp. 5429 (2011)

8. G. P. Agrawal, Nonlinear Fiber Optics, 3rd. ed., (Academic Press, Boston, (2001)

9. Marburger J.H., Prog. Quant. Electr., Vol. 4, pp. 35-1 10. Pergamon Press (1975) 